\title{
Transformaciones en los atributos valorados. Flexibilización laboral, competencias laborales y desplazamiento del actor sindical en Argentina
}

Diego Álvarez Newman ${ }^{1}$

\footnotetext{
1 Licenciado en Sociología, y Doctor en Ciencias Sociales por la Universidad de Buenos Aires (UBA). Investigador del CONICET en el Instituto de Estudios Sociales en Contextos de Desigualdades (IESCODE) de la Universidad de José C. Paz (UNPAZ). Correo electrónico: diegonewman@ hotmail.com
}

Fecha de recepción: 28/11/2019. Fecha de aceptación: 24/08/2020. 


\section{Transformaciones en los atributos valorados. Flexibilización laboral, competencias laborales y desplazamiento del actor sindical en Argentina}

\section{RESUMEN}

El objetivo de este artículo es abordar las transformaciones en los atributos valorados de los trabajadores, a partir del análisis de las políticas basadas en competencias laborales. La hipótesis que se pone en discusión es que el paradigma de las competencias facilita implementar modelos de gestión individualizada del trabajo. En estos son relevantes las habilidades blandas, y estas modalidades de gestión desplazan a los sindicatos de la definición de los atributos valorados. La metodología es cualitativa, y las fuentes de datos utilizadas fueron documentos de organismos nacionales e internacionales encargados de implementar políticas de competencias laborales en Argentina. Asimismo, se revisaron convenios colectivos de trabajo del sector automotriz y entrevistas en profundidad a trabajadores y a líderes de grupo de una terminal automotriz de origen japonés.

Palabras clave: flexibilización laboral, competencias laborales, sindicatos.

Transformations in valued attributes. Labor flexibility, labor competencies and displacement of unions as actors in Argentina

\section{Abstract}

The purpose of this article is to address the transformations in the valued attributes of workers, through the analysis of policies based on labor competencies. The hypothesis is that the competences paradigm facilitates the implementation of individualized work management models, in which soft skills take relevance. These management modalities displace unions from participating in the definition of valued attributes. We will use a qualitative methodology. The data sources were documents from national and international organizations in charge of implementing labor competencies policies in Argentina, and collective labor agreements in the automotive sector. In addition, in-depth interviews were used with workers and group leaders from a Japanese automotive terminal.

Keywords: labor flexibility, labor competencies, unions. 


\section{INTRODUCCIÓN}

El proceso de «modernización empresarial» que se llevó a cabo en Argentina a partir de la década de 1990 se caracterizó por la búsqueda de un aumento de la productividad y de la competitividad mediante la concentración y la implementación de políticas regulatorias tendientes a flexibilizar las actividades productivas y el trabajo.

En este artículo se retoman aspectos que fueron abordados en otro trabajo (2018) con el propósito de profundizar el conocimiento de los procesos de cambio en el mundo del trabajo. En ese marco, se plantea que las estrategias de flexibilización laboral que se implementaron desde entonces fueron centrales para producir transformaciones en los atributos valorados de los sujetos que trabajan. El objetivo del artículo es analizar estas transformaciones a partir del análisis de las políticas basadas en competencias laborales.

Se parte de la hipótesis de que el paradigma de las competencias laborales facilitó la implementación de modelos de gestión individualizada del trabajo, en los cuales toman relevancia las habilidades blandas. A su vez, estos modelos de gestión se posicionan en las áreas de recursos humanos de las empresas, desplazando al actor sindical de la definición de los atributos valorados.

La metodología utilizada es cualitativa y se utilizaron dos técnicas: a) el análisis documental de organismos nacionales e internacionales encargados de implementar políticas de competencias laborales en Argentina, y el análisis de convenios colectivos de trabajo del sector automotriz. Las fuentes de datos de los organismos internacionales fueron documentos de la Organización Internacional de Estandarización (ISO, por su sigla en inglés) — puntualmente la serie de normas ISO 9000—, y del Programa de Cooperación Iberoamericana para el Diseño de la Formación Profesional (IBERFOP) de la Organización de Estados Iberoamericanos (OEI). Con respecto a los actores nacionales, se tomaron fuentes de datos documentales del Programa de Formación y Certificación de Competencias Laborales implementado por el Ministerio de Trabajo, Empleo y Seguridad Social (MTEySS) de la República Argentina; y b) entrevistas en profundidad a trabajadores y a líderes de grupo de una terminal automotriz de origen japonés. Estas fueron realizadas durante el año 2012 en el marco del trabajo de campo llevado a cabo para la tesis doctoral y retomadas en el ańo 2017 en el marco de un proyecto de investigación perteneciente al Programa de Estudios Críticos sobre el Movimiento Obrero (PECMO) del Centro de Estudios e Investigaciones Laborales (CEIL) del Consejo Nacional de Investigaciones Científicas y Técnicas $(\text { CONICET) })^{2}$. En este proyecto se retomaron las indagaciones sobre la gestión por competencias y las subjetividades laborales

\footnotetext{
2 Proyecto UBACyT «Corporaciones, formación de subjetividades y respuestas sindicales: la concreción del orden global en contextos productivos y extraproductivos» (programación 2017-2020).
} 
en corporaciones empresariales, con el objetivo de indagar en las percepciones de los trabajadores acerca de los atributos valorados por la empresa. Se retomaron las entrevistas realizadas a cinco trabajadores pertenecientes a los siguientes sectores: dos «Team members» del sector «ensamblado» de la línea de producción, un «Team leader» del sector «ensamblado» de la línea de producción, un «Team member» del sector «Calidad de proveedores» $\mathrm{y}$ un «Team member» del sector «Mantenimiento». Todos ellos trabajadores de la planta industrial localizada en Zárate, Provincia de Buenos Aires. El criterio de selección de los entrevistados fue que estos contaran con una antigüedad mayor a siete años en la planta industrial, con el fin de comprender la valoración de la experiencia con respecto a otros atributos.

El análisis de las políticas basadas en competencias laborales y las transformaciones en los atributos valorados que estas motorizan se despliegan en tres apartados. En el primero se contextualizan las estrategias de flexibilización laboral en Argentina, que posibilitaron la emergencia de las políticas de competencias laborales. En el segundo apartado se abordan las herramientas de individualización que despliega la gestión por competencias. En el tercero, se analiza la importancia de las "competencias blandas» en la redefinición de los atributos valorados de los sujetos trabajadores.

\section{LA «MODERNIZACIÓN EMPRESARIAL» Y LA FLEXIBILIZACIÓN LABORAL}

La modernización empresarial es un complejo proceso de cambios técnicos, organizacionales y político-laborales que se dan en las empresas que apuntan a metas de competitividad mediante el incremento de la productividad, la reducción de costos de producción, la calidad de los productos y la flexibilización productiva (Dombois y Pries, 1993, p. 14). Este conjunto de transformaciones estratégicas comenzó a plantearse a nivel global a partir de la crisis de mediados de la década de 1970. A su vez, fueron modificando el orden social preexistente mediante la readaptación de los sujetos trabajadores a las nuevas reglas de la estrategia modernizante (Figari, 2001).

Este proceso de transformaciones asumió sus particulares formas en un país que se caracteriza por la inestabilidad crónica como la Argentina. En este país el proceso de modernización empresarial se realizó a través de reformas estructurales luego del proceso hiperinflacionario de los ańos 1989-1990. Sin embargo, cabe destacar que las condiciones necesarias para implementar las transformaciones «modernizantes» comenzaron a imponerse durante la dictadura militar de 1976-1983. Esta dictadura dio inicio a un proceso de desindustrialización que comenzó a desmontar los planes de industrialización sustitutiva de importaciones (ISI) que implementaron los diferentes gobiernos durante el periodo 1945-1975. Asimismo, llevó a cabo un 
proceso de disciplinamiento social que debilitó a los actores sindicales, generando las condiciones para comenzar a desmontar lo que las élites locales consideraban como una «actividad industrial ineficiente» (Villarreal, 1985).

Los cambios estructurales se produjeron luego de la adhesión al Consenso de Washington durante el gobierno de Carlos Menem (1989-1999), de fuerte sesgo neoliberal. El programa de reconversión productiva que se llevó a cabo en este marco podría resumirse en dos ejes: 1) el aumento de la productividad y de la competitividad mediante la concentración, es decir, la supervivencia de las industrias consideradas «eficientes» en detrimento de las pequeñas y medianas empresas no competitivas frente a la apertura comercial (Schor, 2002); y 2) la promulgación de leyes y decretos junto a la implementación de políticas regulatorias tendientes a flexibilizar las actividades productivas y el trabajo (Neffa, 2014).

En este marco, comprender la flexibilización laboral es de suma importancia, porque es la principal estrategia que utilizan las empresas concentradas para mejorar el consumo productivo de la fuerza de trabajo. Esta estrategia es propia de lo que se denomina "management posfordista" para desestructurar todos aquellos aspectos del trabajo que puedan constituir limitaciones al incremento de la productividad y la reducción de costos.

Los inicios de la estrategia de flexibilización laboral pueden ubicarse con la aprobación de la Ley nacional de empleo 24013/91, que la institucionalizó y fue el punto de partida de la primera oleada que se extendió durante toda la década de 1990 hasta la salida de la convertibilidad (2002) ${ }^{3}$. Durante todo ese periodo se avanzó en una batería de leyes, decretos y políticas tendientes a generalizar la inestabilidad laboral y la desprotección del trabajo. Se avanzó sobre los marcos de la negociación colectiva, los salarios, las condiciones y las jornadas de trabajo, el derecho a huelga, las indemnizaciones, e incluso se crearon instrumentos financieros no remunerativos como los tickets canasta, que funcionaron como un complemento al salario (Boyer y Neffa, 2004).

La crisis de 2001-2002, el grado de organización que habían mostrado los movimientos de trabajadores desocupados y el nivel de conflictividad no dieron margen para la continuidad de las políticas de ajuste y flexibilización. La devaluación de 2002 significó la salida del régimen de la convertibilidad y sentó las condiciones para que a partir de 2003 la economía argentina—y en particular el sector manufacturero- inicie una senda de expansión (Azpiazu y Schorr, 2010).

3 La Ley de Convertibilidad 23.928, fue decretada el 27 de marzo de 1991 por el Congreso de la República Argentina durante el gobierno de Carlos Menem, bajo la iniciativa del entonces ministro de Economía Domingo Cavallo. Estuvo vigente durante casi once ańos, hasta que fue derogada el 6 de enero de 2002. Esta ley establecía la paridad del peso argentino con el dólar estadounidense. 
Durante el periodo 2003-2010 se produjo un notorio avance en la creación de empleo y un gran dinamismo y expansión de la negociación colectiva, mejorando notoriamente las condiciones salariales de los trabajadores ${ }^{4}$. Estos avances posibilitaron un proceso de revitalización sindical (Senén Gonzalez y Del Bono, 2013; Lenguita, 2011) que motivó un reflujo de la estrategia flexibilizante. Sin embargo, se mantuvieron activas las normas flexibilizantes de la década anterior en cuanto a los aspectos vinculados a la inestabilidad laboral y las condiciones de trabajo (Delfini y otros, 2013). Si se toma la totalidad del ciclo kirchnerista 2003-2015, este podría ser caracterizado como un periodo de revitalización del trabajo, pero con persistencia de la flexibilización laboral.

Las condiciones políticas para la segunda oleada de la estrategia de flexibilización laboral se produjeron en el año 2015 con la llegada de la coalición "Cambiemos al Gobierno». La certeza de que se iba a promover transformaciones tendientes a desestructurar los avances logrados en la década anterior en materia laboral no se hizo esperar. En el informe «El estado del Estado», publicado por el Gobierno Nacional en junio de $2016^{5}$ pueden encontrarse los argumentos que intentan legitimar la necesidad de avanzar en un nuevo proceso de flexibilización laboral. Con la llegada del macrismo al poder se abrió una segunda oleada, y sus límites y alcances están aún en disputa. En esta segunda oleada se volvió a incentivar la flexibilización laboral desde el Estado, mediante la promoción de reformas laborales regresivas similares a las de la década de $1990^{6}$.

\footnotetext{
4 Durante el periodo 2003-2010 se crearon 3,1 millones de puestos de trabajo. La tasa de empleo en el año 2002 era del 33,3\% mientras que para el año 2010 ascendió al 42,5\%. Por su parte, la tasa de desocupación abierta era del $17,8 \%$ en 2002 y para el año 2007 ya era de 7,5\%.

5 Este informe, elaborado por la coalición gobernante durante el periodo 2015-2019, pretende mostrar los errores y excesos cometidos por el gobierno kirchnerista en la administración pública. El documento se posicionó como una fuente argumental de denuncia al «populismo».

6 Durante 2017 y 2018, luego de haber triunfado en las elecciones parlamentarias y en el marco de las negociaciones y los acuerdos con el FMI, el gobierno nacional envió al parlamento tres proyectos de reforma laboral que fueron rechazados por la dirigencia sindical y la oposición política. Si bien no entraron en vigencia, estos proyectos permitieron conocer la agenda del capital concentrado en materia de las regulaciones del trabajo. Por un lado, retomaron e intentaron profundizar e instituir aspectos flexibilizantes tradicionales, como la vuelta de las pasantías, la creación de bancos de horas, las reducciones en los cálculos de las indemnizaciones e impedimentos a las y los trabajadores tercerizados para hacerle juicio a la «empresa madre». Por otro lado, los proyectos presentaron aspectos flexibilizantes «originales» como la institucionalización del «Fondo de cese laboral» (que ya se venía implementando en el sector de la construcción), en el que las y los trabajadores aportarían a su propio despido; y la creación de la figura del «trabajador profesional autónomo económicamente vinculado», que mediante un estatuto particular intentaría regular el universo de los monotributistas, cuyos ingresos mayoritarios provienen de un solo contratista.
} 


\section{LOS MARCOS DE LA GESTIÓN INDIVIDUALIZADA}

En este apartado se sostiene que la consolidación de la flexibilización laboral desde los años noventa proporcionó las condiciones y facilitó la implementación de modelos de gestión individualizada del trabajo a través del paradigma de las competencias laborales.

Una vez instituidos los marcos legales de la flexibilización, diversos organismos internacionales comenzaron a brindar asistencia técnica y financiamiento para acercar a las empresas y a los actores del mundo del trabajo perspectivas que permitan abordar las relaciones laborales de una manera «moderna». Estas perspectivas pretendían transformar un modelo que era considerado «rígido» y propio de la división sociotécnica del paradigma de la administración científica del trabajo (Spinosa, 2006).

Organismos como el Centro Interamericano para el Desarrollo del Conocimiento en la Formación Profesional de la Organización Internacional del Trabajo (CINTERFOR/OIT), el Fondo Multilateral de Inversiones del Banco Interamericano de Desarrollo (FOMIN/BID) y el Programa de Cooperación Iberoamericana para el Diseño de la Formación Profesional (IBERFOP/OEI) fueron determinantes para difundir e instrumentar los enfoques modernizantes en América Latina. Particularmente en Argentina, estos organismos tuvieron una gran influencia en la implementación de programas basados en competencias laborales, al punto que sus recomendaciones se constituyeron en ejes prioritarios de las políticas públicas en materia de formación profesional. Este enfoque tomó forma en las políticas laborales recién en los inicios del siglo XXI, cuando las reformas flexibilizantes ya estaban consolidadas.

Las políticas laborales basadas en competencias se canalizaron a través del Ministerio de Trabajo, Empleo y Seguridad Social de la Nación (MTEySS) mediante la modalidad de un Programa de Formación y Certificación de Competencias Laborales (PFCCL).

Este programa surgió con el propósito de establecer las bases institucionales y metodológicas para desarrollar un sistema nacional de certificaciones. En un primer momento, entre los años 2001 y 2004, se realizaron las experiencias piloto en formación y certificación en cuatro sectores de la actividad. Durante esta experiencia, el MTEySS ofició de veedor del Programa, CINTERFOR/OIT realizó la asistencia técnica junto al IBERFOP/OEI, y FOMIN/BID otorgó el financiamiento. Posteriormente comenzó el proceso de transferencia del programa de los organismos internacionales hacia el MTEySS y en 2006 quedó totalmente transferido. Hasta el año 2019 se crearon y se registraron normas de competencia laboral en roles ocupacionales de treinta sectores de la actividad. 
En su fundamentación, el programa hace especial hincapié en la necesidad de implementar el enfoque de las competencias para la formación profesional, planteando la existencia de «un contexto de incertidumbre y constantes cambios tecnológicos y organizacionales [...] y en una economía globalizada». Dicho contexto requeriría una readaptación de las capacidades laborales de los trabajadores: «En el caso del mundo del trabajo, las competencias son aquellas capacidades que permiten a los individuos establecer estrategias cognitivas y resolutivas en relación con los problemas que se les presentan en el ejercicio de sus roles laborales. Las normas de competencia pretenden ser descriptores densos de estas habilidades, conocimientos y criterios de actuación» (Ministerio de Trabajo, Empleo y Seguridad Social, s/f).

La definición de competencias laborales adoptada en el marco del programa muestra cuatro dimensiones en la relación entre los sujetos y el trabajo. Las aptitudes («saber hacer») son las que movilizan las capacidades vinculadas a los conocimientos y las destrezas técnicas; las actitudes («saber ser») movilizan las capacidades para operar en situaciones problemáticas y de incertidumbre; las normas de competencia describen las capacidades y criterios necesarios de actuación y adecuación; y los roles laborales hacen referencia al papel que cumplen los trabajadores en el espacio de trabajo en relación con las normas de competencia (Álvarez Newman y Hirsh, 2011).

Siguiendo a Zariffian (1999, p. 34) las competencias hacen referencia principalmente a las capacidades que le permiten a un individuo tomar la iniciativa y responsabilizarse con éxito ante una situación profesional en función de la misión o de los objetivos que le fueron confiados. Los documentos analizados del MTEySS y de los organismos internacionales que participan del armado de las políticas de formación profesional muestran esta relevancia de la dimensión del saber ser.

Desde el punto de vista de la organización de los procesos de producción, se asiste a una revalorización del capital humano; el perfil de trabajador que se demanda también cambia; exigiéndose un conjunto de nuevas competencias [...]. Se requiere que la persona sea capaz de adaptarse y promover una nueva forma de organización del trabajo, caracterizada por estructuras menos jerarquizadas y que desarrolle actividades variadas y diferenciadas. De este modo, la responsabilidad del trabajador ya no se circunscribe a ejecutar una acción permanente y rutinaria, sino que consiste además en analizar el proceso con un enfoque integral, para lograr la mejora continua del mismo y su adaptación al cambio. Entre las nuevas formas de organización del trabajo, cabe destacar la ampliación y enriquecimiento de tareas, la polivalencia de la mano de obra, la organización modular y los grupos semiautónomos de trabajo. Asimismo, es cada vez más frecuente el involucramiento de los trabajadores en el control de calidad y la planificación del trabajo, en la integración de equipos, en el mantenimiento preventivo del equipo, 
en el diagnóstico y solución de problemas de la producción y en la rotación de tareas (Programa de Cooperación Iberoamericana para el Diseño de la Formación Profesional, 2000, pp. 11-12).

Los atributos valorados ponen el foco principalmente en competencias blandas, tales como la adaptación, la implicación, la autonomía y la responsabilización. Claramente, este enfoque intenta rastrear nuevas fuentes de productividad en el nivel subjetivo, más allá de la clásica concepción de la fuerza de trabajo.

La identificación de las competencias blandas es el puntal que permite gestionar el trabajo de manera individualizada. Ahora bien, ¿cómo deben gestionarse esas competencias? La serie de normas ISO 9000 de gestión de la calidad, que promueve normas estandarizadas para la gestión de los recursos humanos en las organizaciones empresariales proporciona el marco preciso sobre el que deben gestionarse estos atributos.

Para aumentar la participación y la motivación de las personas que la integran, la organización debería considerar actividades tales como:

- desarrollar un proceso para compartir el conocimiento y utilizar la competencia de las personas, por ejemplo, un esquema para recopilar ideas para la mejora;

- introducir un sistema de reconocimiento y recompensa adecuado, basado en evaluaciones individuales de los logros de las personas;

- establecer un sistema de calificación de habilidades y planes de carrera, para promover el desarrollo de las personas;

- revisar continuamente el nivel de satisfacción y las necesidades y expectativas de las personas, $\mathrm{y}$

- proporcionar oportunidades para realizar tutorías y adiestramiento profesional (coaching) (Organización Internacional de Estandarización, 2009, p. 7).

Las herramientas de gestión de los RRHH que sitúan los ejes planteados en la norma internacional son: la implementación de círculos de calidad (o grupos de mejora), la introducción de un sistema que permita identificar y recompensar por sus atributos a los sujetos meritorios, el establecimiento de niveles que proporcionen un plan de carrera de acuerdo a los atributos de las personas, las evaluaciones de desempeño y la formación continua. Estas herramientas en su conjunto permiten individualizar e indagar en posibles fuentes subjetivas para el mejoramiento de la productividad, relacionadas a los atributos de las personas en relación a los objetivos de la organización.

\section{LAS COMPETENCIAS BLANDAS Y EL DESPLAZAMIENTO DEL ACTOR SINDICAL}

En este apartado se complejizan las formas que asumieron los modelos de gestión individualizada del trabajo a través del paradigma de las competencias laborales. 
Este paradigma impulsó fuertes cambios en los atributos valorados de los sujetos trabajadores. Por un lado, se produjeron redefiniciones polivalentes que reconvirtieron a los puestos de trabajo en «roles» laborales. Estas redefiniciones fueron otorgando centralidad a las habilidades blandas como el compromiso y la responsabilización para ejercer diferentes roles. Por otro lado, se resalta que los actores centrales de estos cambios fueron las áreas de recursos humanos, en detrimento del actor sindical.

La gestión por competencias laborales establece un sistema de diferenciaciones sobre la base de las competencias blandas de las personas, y promueve la individualización y una particular forma de distinción entre los trabajadores.

El patrón de diferenciación anterior al de las competencias laborales fue el sistema de las calificaciones profesionales, que en el marco del modelo taylorista fordista buscaba fijar a la fuerza de trabajo en sus puestos. Este era un sistema de clasificación profesional que establecía rangos y jerarquías y se instituía en las categorías laborales negociadas en los convenios colectivos de trabajo. Una de las cuestiones más importantes de este paradigma es que esos rangos y jerarquías profesionales diferencian a los trabajadores a partir de categorías laborales. Esta forma de diferenciación sufre a su vez segmentaciones por la antigüedad, que es un indicador clave que valora la experiencia.

Este tradicional sistema de clasificación profesional fue característico de las rondas de negociación colectiva anteriores al año 1994 en Argentina, específicamente de las rondas de 1975 y las de 1988-1989) (Battistini, 2000). Los convenios colectivos de trabajo (en adelante CCT) firmados en esas rondas se caracterizaban por una gran cantidad de categorías, que contenían cada una de ellas las descripciones de las tareas a realizar. Por ejemplo, el CCT 8/89, firmado entre el Sindicato de Mecánicos y Afines del Transporte Automotor (SMATA) ${ }^{7}$ y Autolatina S.A. ${ }^{8}$ en la ronda de 1989 contaba con diez categorías para la producción de automotores, con un encuadramiento de tareas para cada una de ellas (art. 6, «Discriminación de categorías laborales y de tareas»).

Por el contrario, el paradigma de las competencias laborales dispone un patrón más flexible. Este patrón se apoya menos en las descripciones sociotécnicas de los puestos para cada categoría y mucho más en las habilidades blandas que permiten la adaptación a variados roles laborales. Una de las tendencias generales son las redefiniciones polivalentes de los puestos de trabajo (Campos y Campos, 2010), transformados en «roles».

\footnotetext{
El SMATA es el sindicato nacional que proporciona el encuadre gremial a los trabajadores del sector automotriz.

8 Autolatina S.A. fue el nombre de la fusión entre las empresas automotrices Ford y Volkswagen durante el periodo 1987-1995 en Argentina y en Brasil
} 
Por supuesto, esas redefiniciones no tomaron la misma forma en los distintos sectores de la actividad. El sector automotriz en Argentina fue paradigmático de estas transformaciones. En los CCT firmados en los ańos posteriores a 1996 entre Toyota Argentina S.A. y el SMATA', la polivalencia y las competencias blandas tales como la implicación con la filosofía corporativa, la participación en los grupos de mejora continua, la comunicación, la responsabilización y el trabajo en equipo se encuentran desplegadas a lo largo de los diferentes artículos que componen estos convenios (Álvarez Newman, 2012). En esos convenios las redefiniciones polivalentes fueron llevadas al extremo. Por ejemplo, en el CCT 730/2005-E ${ }^{10}$ las categorías se redujeron a dos: «team member» $\mathrm{y}$ «team leader» expresando sus contenidos una polivalencia total.

Lo que emerge, también como tendencia general desde los años 90, es que las bases sociotécnicas de los puestos de trabajo desplegadas en grandes cantidades de categorías en los CCT fueron perdiendo peso y los atributos valorados se fueron desplazando hacia las habilidades blandas.

Las nuevas formas de gestionar las diferenciaciones y valorar los atributos laborales se estipulan principalmente en las herramientas que permiten identificar las capacidades individuales para ejercer los roles laborales y adaptarse a las demandas de la empresa. Los CCT generalmente fijan las escalas salariales y ciertas condiciones laborales, pero ya no definen las características sociotécnicas de los puestos de trabajo y sus perfiles. Incluso, para el paradigma de las competencias lo trascendente no son los puestos sino los «roles laborales».

Esta forma de diferenciación y de individualización hace que las organizaciones empresariales definan cuáles son los atributos valorados teniendo en cuenta la base de competencias blandas con las que cuentan los sujetos trabajadores.

Las áreas de recursos humanos de las empresas comenzaron a ser un actor central, dado que son estas las que definen los atributos valorados sin la participación de los sindicatos. Las áreas de recursos humanos ya no son esas «oficinas de personal» que solamente liquidan sueldos y fiscalizan el cumplimiento de lo pactado en los CCT, sino que son las que gestionan el valor y el reconocimiento de cada persona en función de pautas de desempeño.

Las pautas y las evidencias de desempeño se definen de manera unilateral en el ámbito de la empresa. Esto reduce fuertemente la incidencia de los sindicatos

\footnotetext{
9 Se hace referencia al convenio colectivo de trabajo 190/1996-E, firmado el 27/5/1996; al 449/2001-E, firmado el 28/12/2000; y al 730/2005-E, firmado el 4/11/2004 entre Toyota Argentina y el SMATA. Estos convenios regulan la actividad laboral de la planta industrial localizada en Zárate, Provincia de Buenos Aires, con 5393 trabajadores (2018).

10 Anexo «B» del art. 35, «Criterios para el traspaso de niveles - Factores considerados al momento de la evaluación».
} 
en los temas vinculados a la organización del trabajo. A través de entrevistas en profundidad a team leaders de Toyota Argentina S.A., identificamos que existen indicadores de desempeño que son considerados como los más importantes por sus vínculos directos con los salarios, como las cantidades de sugerencias que deben proponer mensualmente, los niveles de participación en los círculos de calidad y los grados de compromiso en las reuniones de trabajo ${ }^{11}$. Al definirse las pautas de desempeño en el nivel de la empresa, es común en la planta industrial de Toyota Argentina que los líderes de grupo ocupen el lugar de los delegados sindicales.

— ¿Y cómo se resuelven esos problemas [con respecto a la intensidad de los ritmos de producción]?

- Nosotros cada vez que tenemos un problema, un inconveniente, vamos al líder, le planteamos y él nos dice. [...] ayer paró la línea porque yo mandé mal algo [...]. Y bueno, agarrate, vienen a taladrarte. Y después en la charla de cinco minutos te dicen 'ensamble paró la línea por un defecto', no nombran a quién pero todos sabemos y todos se empiezan a reír como diciendo: 'boludo'. Y bueno, que eso no tiene que pasar, entonces como contramedida te ponen que antes de armar tenés que mirar el manifiesto que es una hoja así que te dice lo que lleva ese modelo (entrevista a Jorge, trabajador de línea de producción de Toyota Argentina).

Es habitual que frente a los problemas que se registran en la producción los trabajadores no recurran a los delegados sindicales. Son los líderes de los grupos los que absorben las demandas de los trabajadores. Hay una serie de instancias que canalizan los planteamientos que atańen a las modalidades de trabajo. En primer lugar, se apela al líder como referencia directa y este los aborda en las reuniones «cortas» de equipo y decide la medida correctiva. Si los problemas son más complejos, se plantean de manera individual bajo el «sistema de sugerencias» o de manera grupal junto al equipo de trabajo en los círculos de control de calidad. Pero la referencia no suele ser el espacio sindical ${ }^{12}$.

La evidencia empírica sugiere un desplazamiento del actor sindical en la participación de la gestión de los atributos valorados, precisamente porque estos ya no

\footnotetext{
11 En la planta industrial de Toyota Argentina se dispone de una serie de herramientas de control que en su conjunto son parte del Sistema Toyota de Producción (STP). Los «sistemas de sugerencias» son mejoras que deben proponer los trabajadores de la planta industrial de manera individual. La empresa exige dos sugerencias mensuales a cada uno de sus empleados. Los círculos de control de calidad son grupos de mejora de la producción. En estos grupos se organizan de siete a diez trabajadores que diseñan un proyecto para mejorar algún aspecto de la producción. Las reuniones de trabajo habitualmente se denominan como "charla de cinco minutos». Se hacen dos al día (al entrar a trabajar y al terminar) y en ellas se conversa sobre los principales problemas que surgieron en la jornada de trabajo. 12 En algunas entrevistas, incluso, los trabajadores de la planta confunden al sindicato con la obra social. Y, en muchas otras ocasiones era asociado a una organización que se remite solamente a negociar pisos salariales.
} 
son negociados en el marco de los CCT sino que son definidos y registrados por las áreas de recursos humanos e instrumentados por referentes de la empresa (los «líderes»). Estos fueron ocupando el rol que habitualmente cumplían los delegados sindicales de base.

\section{REFLEXIONES FINALES}

Tomando en cuenta que el objetivo de este artículo ha sido abordar las transformaciones en los atributos valorados de los sujetos que trabajan a partir del análisis de las políticas basadas en competencias laborales, y que se partió de la hipótesis de que el paradigma de las competencias facilita la implementación de modelos de gestión individualizada del trabajo en los que toman relevancia las habilidades blandas, interesa resaltar, en primer lugar, que la gestión por competencias requirió de un proceso de flexibilización laboral previo para poder implementarse. Es decir, la preeminencia que otorgaba el modelo de las calificaciones a fijar a la fuerza de trabajo en los puestos no daba lugar a pensar en la necesidad de establecer pautas sobre la base de competencias blandas. Es precisamente la necesidad de polivalencia la que redefine a los puestos de trabajo en roles laborales y la que lleva a las empresas a indagar en las competencias blandas de las que dispongan a los sujetos para ejercer roles diversos.

Se configura así un esquema de gestión individualizada de los atributos, que entra en contradicción con la convención colectiva de trabajo. La pérdida de poder de los sindicatos no solo se visualiza en los CCT a través de la aceptación de la polivalencia, sino también a través de la falta de definición de las competencias blandas valoradas y las pautas de desempeño. Los sindicatos no participan de esas definiciones, dado que se gestionan desde las áreas de recursos humanos.

Frente a la ausencia de estas referencias, los delegados sindicales de base fueron perdiendo incidencia en el control de la organización del trabajo, dado que son los team leaders los que guían a los trabajadores acerca de los atributos necesarios para lograr las mejoras salariales.

\section{REFERENCIAS}

Álvarez Newman, D. (2012). Organización del trabajo y dispositivos de control en el sector automotriz: el toyotismo como sistema complejo de racionalización. Trabajo y Sociedad 18, vol. XV.

Álvarez Newman, D. (2018). La hegemonía del capital: estudio sobre el dispositivo de implicación en el trabajo. Buenos Aires: Facultad de Ciencias Sociales - Universidad de Buenos Aires / Teseo. 
Álvarez Newman, D. y Hirsch, D. (2011). La formación por competencias en el servicio de mantenimiento y reparación de automotores: El rol del SMATA en el proceso de normalización de trabajadores. $2^{\circ}$ Congreso Internacional de Relaciones del Trabajo de la Universidad de Buenos Aires.

Azpiazu, D. y Schorr, M (2010). La industria argentina en la posconvertivilidad: reactivación y legados del neoliberalismo. Problemas del desarrollo, revista latinoamericana de economía, 41(161). https://doi.org/10.22201/iiec.20078951e.2010.161.18498

Battistini, O. (2000). «La negociación colectiva y la estructura sindical argentina (1988-1998)». Tesis de maestría en Ciencias Sociales del Trabajo. Centro de Estudios Avanzados, Universidad de Buenos Aires, Buenos Aires, Argentina.

Campos, J. y Campos, L (2010). Acerca de la persistencia de cláusulas de flexibilización laboral en los convenios colectivos de trabajo homologados en la posconvertibilidad. Razón y Revolución, 20.

Convenio Colectivo de Trabajo 8/89 firmado entre el Sindicato de Mecánicos y Afines del Transporte Automotor (SMATA) y Autolatina SA el 8 de junio de 1989; Buenos Aires.

Convenio Colectivo de Trabajo 190/1996-E firmado entre el Sindicato de Mecánicos y Afines del Transporte Automotor (SMATA) y Toyota Argentina SA el 27 de mayo de 1996; Buenos Aires.

Convenio Colectivo de Trabajo 449/2001-E firmado entre el Sindicato de Mecánicos y Afines del Transporte Automotor (SMATA) y Toyota Argentina SA el 28 de diciembre de 2000; Buenos Aires.

Convenio Colectivo de Trabajo 730/2005-E firmado entre el Sindicato de Mecánicos y Afines del Transporte Automotor (SMATA) y Toyota Argentina SA el 04 de noviembre de 2004; Buenos Aires.

Delfini, M., Drolas, A. y Medina, J (2013). Continuidades y rupturas en las relaciones laborales de Argentina tras la crisis del neoliberalismo; Observatorio Laboral Revista Venezolana Vol. 6, No 11, Universidad de Carabobo, Venezuela.

Dombois, R. (1993). Modernización empresarial y cambios en las relaciones industriales de América Latina y Europa. En Ludger Pries (ed.), Modernización empresarial: tendencias en América Latina y Europa. Buenos Aires: Nueva Sociedad - Friedrich Ebert.

Figari, C. (2001). Lógicas de formación y de calidad en la modernización empresarial. Estudios del Trabajo, 22, 95-120.

Lenguita, P. (2011). Revitalización desde las bases del sindicalismo argentino. Nueva Sociedad, 232, 137-149.

Ministerio de Trabajo, Empleo y Seguridad Social (s/f). El marco conceptual de la normalización y certificación de competencias laborales: documento elaborado en el marco del Programa de Formación y Certificación de Competencias Laborales. Argentina: Programa de Calidad del Empleo y la Formación Profesional - Unidad Técnica de Certificación de Competencias / MTEySS.

Neffa, J.C. (2004). La forma institucional relación salarial y su evolución en la Argentina desde una perspectiva de largo plazo. En R. Boyer y J.C. Neffa (eds.), La economía 
argentina y sus crisis (1976-2001). Visiones institucionalistas y regulacionistas (pp. 105149). Buenos Aires: Miño y Dávila/CEIL-PIETTE/CONYCET.

Neffa, J.C. (2014). Subcontratación, tercerización y precarización del trabajo y el empleo: una visión regulacionista desde la economía del trabajo y el empleo. En J.C. Ospina (coord.), La subcontratación laboral en América Latina: miradas multidimensionales (pp. 69-97). Medellín: Escuela Nacional Sindical / CLACSO.

Organización Internacional de Estandarización (2009). Norma ISO 9004. Gestión para el éxito sostenido de una organización. Ginebra: ISO.

Programa de Cooperación Iberoamericana para el Diseño de la Formación Profesional de la Organización de Estados Iberoamericanos (2000). Análisis ocupacional y funcional del trabajo. Madrid: IBERFOP / Consejo de Normalización y Certificación de Competencia Laboral (CONOCER).

Schorr, M. (2002). Mitos y realidades del pensamiento neoliberal: la evolución de la industria manufacturera argentina durante los años noventa. En AAVV, Más allá del pensamiento único. Hacia una renovación de las ideas económicas en América Latina. Buenos Aires: CLACSO.

Senén González, C. y Del Bono, A. (comp.) (2013). La revitalización sindical en Argentina: alcances y perspectivas. Buenos Aires: Universidad Nacional de La Matanza / Prometeo.

Spinosa, M. (2006). Los saberes y el trabajo. Anales de la Educación Común, 2(5), 164-173. Villarreal, J. (1985). Los hilos sociales del poder. En Eduardo Josami y otros, Crisis de la dictadura argentina. Política Económica y cambio social (pp. 201-272). Buenos Aires: Siglo XXI.

Zarifian, F. (1999). El modelo de competencia y los sistemas productivos. Montevideo: CINTERFOR/OIT. 